Braz J Med Biol Res, October 2011, Volume 44(10) 1018-1024

doi: 10.1590/S0100-879X2011007500105

\section{Typical and atypical enteropathogenic Escherichia coli bacterial translocation associated with tissue hypoperfusion in rats}

A.M.A. Liberatore, F.C. Moreira, T.A.T. Gomes, J.L. Menchaca-Diaz and I.H.J. Koh

The Brazilian Journal of Medical and Biological Research is partially financed by

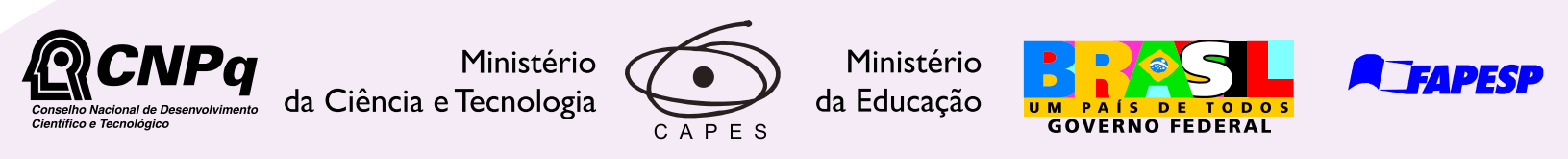

Institutional Sponsors
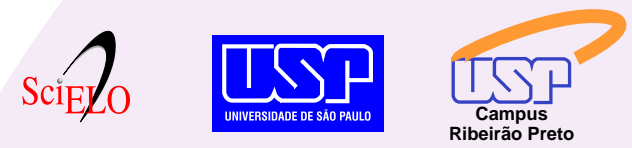

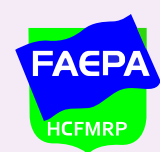

๑ SHIMADZU

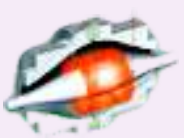

Explore High - Performance MS Orbitrap Technology In Proteomics \& Metabolomics

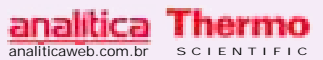




\title{
Typical and atypical enteropathogenic Escherichia coli bacterial translocation associated with tissue hypoperfusion in rats
}

\author{
A.M.A. Liberatore ${ }^{1 *}$, F.C. Moreira ${ }^{2 *}$, T.A.T. Gomes ${ }^{2}$, \\ J.L. Menchaca-Diaz ${ }^{1}$ and I.H.J. Koh ${ }^{3}$ \\ ${ }^{1}$ Departamento de Pediatria, ${ }^{2}$ Departamento de Microbiologia, Immunologia e Parasitologia, \\ ${ }^{3}$ Departamento de Cirurgia, Escola Paulista de Medicina, Universidade Federal de São Paulo, \\ São Paulo, SP, Brasil
}

\begin{abstract}
Although enteropathogenic Escherichia coli (EPEC) are well-recognized diarrheal agents, their ability to translocate and cause extraintestinal alterations is not known. We investigated whether a typical EPEC (tEPEC) and an atypical EPEC (aEPEC) strain translocate and cause microcirculation injury under conditions of intestinal bacterial overgrowth. Bacterial translocation (BT) was induced in female Wistar-EPM rats (200-250 g) by oroduodenal catheterization and inoculation of $10 \mathrm{~mL} 10^{10} \mathrm{colony}$ forming unit (CFU)/mL, with the bacteria being confined between the duodenum and ileum with ligatures. After $2 \mathrm{~h}$, mesenteric lymph nodes (MLN), liver and spleen were cultured for translocated bacteria and BT-related microcirculation changes were monitored in mesenteric and abdominal organs by intravital microscopy and laser Doppler flow, respectively. $\mathrm{tEPEC}(\mathrm{N}=11)$ and aEPEC $(\mathrm{N}=11)$ were recovered from MLN $(100 \%)$, spleen $(36.4$ and $45.5 \%)$, and liver ( 45.5 and $72.7 \%)$ of the animals, respectively. Recovery of the positive control E. coli R-6 $(\mathrm{N}=6)$ was $100 \%$ for all compartments. Bacteria were not recovered from extraintestinal sites of controls inoculated with non-pathogenic $E$. coli strains HB101 $(\mathrm{N}=6)$ and $\mathrm{HS}(\mathrm{N}=10)$, or saline. Mesenteric microcirculation injuries were detected with both EPEC strains, but only aEPEC was similar to E. coli R-6 with regard to systemic tissue hypoperfusion. In conclusion, overgrowth of certain aEPEC strains may lead to BT and impairment of the microcirculation in systemic organs.
\end{abstract}

Key words: Enteropathogenic Escherichia coli (EPEC); Atypical EPEC; Bacterial translocation; Microcirculation; Tissue perfusion

\section{Introduction}

Bacterial translocation (BT) is defined as the migration of indigenous bacteria as well as their products from the intestinal lumen to the mesenteric lymph nodes and to other extraintestinal sites (1). Gut bacterial overgrowth, intestinal barrier disruption, and immune deficiency conditions have been proposed as the three major BT-inducing factors (2).

According to a growing consensus, under physiological conditions, BT occurs regularly for intestinal microbe presentation and this event has been referred to as the gut immune enhancing mechanism (3). The experimental and clinical demonstrations of this phenomenon are based on mesenteric lymph nodes (MLN) showing positive cul- ture. However, in critically ill patients, such as patients in shock, with sepsis and burns, BT seems to promote the exacerbation of inflammatory responses, attributed to the crosstalk between the gut and systemic immunity through the lymphatic route communication by carrying gut-derived pro-inflammatory products to the bloodstream $(4,5)$.

We have previously identified an indigenous naturally tetracycline-resistant Escherichia coli strain (E. coli R-6, serotype ONT:H2) in the mesenteric lymph nodes and Peyer's patches of a rat, after 10 days of antibiotic therapy (6). At high concentrations ( $10^{10}$ colony forming unit (CFU)/ $\mathrm{mL}$ ), E. coli R-6 confined in the small bowel of rats caused consistent and substantial translocation to MLN, liver and

Correspondence: I.H.J. Koh, Departamento de Cirurgia, EPM, UNIFESP, Rua Pedro de Toledo, 781, 9a andar, 04039-032 São Paulo, SP, Brasil. E-mail: ivankoh@terra.com.br

*These authors contributed equally to this study.

Received August 23, 2010. Accepted July 27, 2011. Available online August 19, 2011. Published October 10, 2011. 
spleen with marked injury to the mesenteric microcirculation (7). Such events lead to tissue hypoperfusion of the gut and abdominal organs and are attributed to the lymphatic route of BT by triggering gut-associated lymphoid tissue (GALT) activation and pro-inflammatory cytokine release into the systemic compartment, resulting in the amplification of the host inflammatory state (8). In addition, when the BT process was associated with non-lethal sepsis, an increased mortality rate was observed (9)

Although commensal E. coli strains are usually nonvirulent, when one or more BT-predisposing factors are present in the host, they can translocate and consequently generate or aggravate an infectious illness $(4,10)$.

Enteropathogenic E. coli (EPEC) is one of the six categories of diarrheagenic $E$. coli (11), divided into typical and atypical EPEC (tEPEC and aEPEC, respectively). Although tEPEC are leading agents of infantile diarrhea in developing countries, they are rarely detected in industrialized countries, where aEPEC seem to be more important diarrheal agents (12-14). By definition, the main difference between tEPEC and aEPEC is the presence of the so-called EPEC adherence factor (EAF) plasmid, which is found only in tEPEC strains $(12,15)$.

The main mechanism of EPEC pathogenesis is attributed to an attaching and effacing (AE) lesion, which is encoded by a chromosomal pathogenicity island called "locus of enterocyte effacement" (LEE) $(11,14)$. Among various other proteins, LEE encodes the outer membrane adhesive protein intimin and its translocated receptor Tir (translocated intimin receptor) $(11,14)$. Besides loss of absorptive microvilli due to the AE lesion (16), it has been shown that the coordinated activities of various proteins secreted by EPEC trigger several other changes in the intestine $(16,17)$, leading to the establishment of diarrhea.

In the present study, we analyzed the potential of both tEPEC and aEPEC to translocate from the intestine and spread into the systemic bloodstream, thus causing systemic alterations under a situation of intestinal bacterial overgrowth.

\section{Material and Methods}

\section{Microbiological procedures}

Bacterial strains. aEPEC strain 1711-4 (serotype O51:H40) isolated from a child with diarrhea in the city of São Paulo, Brazil (1989) and tEPEC prototype strain E2348/69 (serotype 0127:H6) also isolated from a child with diarrhea were used. Laboratory strain E. coliHB101 and E. coli strain HS (serotype O9:H4), previously isolated from a healthy human and which colonize the human gastrointestinal tract without producing any apparent clinical symptoms (18), were used as non-pathogenic controls. The rat strain $E$. coli R-6 (serotype ONT:H2), with the ability to translocate (6), was used as a positive BT control. This strain is devoid of the main virulence genes of the diarrheagenic $E$. colicategories, including the EPEC eae gene (Vieira MM, Liberatore AMA,
Koh IHJ, Gomes TAT, unpublished data).

Bacterial growth. Strains were cultured in tryptic soy broth or Luria-Bertani broth (Difco, USA). MacConkey agar (Difco) and tryptic soy agar (Difco) were used as solid media. Incubations were carried out for 18 to $20 \mathrm{~h}$ at $37^{\circ} \mathrm{C}$.

Polymerase chain reaction $(P C R)$ analysis. Prior to the BT assays, feces were collected from all animals and cultivated on MacConkey agar plates. After overnight incubation at $37^{\circ} \mathrm{C}$, a portion of each of the resulting enriched bacterial confluent growth was collected with a loop, suspended in saline, and boiled. This suspension was used in PCR to assess the presence of the EPEC eae gene (encoding intimin) as previously reported (19). Amplification reactions were performed with $0.2 \mathrm{mM}$ dNTP, $1.5 \mathrm{mmol} \mathrm{MgCl}_{2}, 2.5 \cup$ Taq DNA polymerase, and $100 \mathrm{pmol}$ of each primer. Amplified products were analyzed on $2 \%$ agarose gels and visualized by ethidium bromide staining. Only animals lacking eae-positive bacteria were used for the BT experiments. This procedure was used in order to avoid the use of animals colonized by AE-producing $E$. coli strains, as well as the AE-producing mouse pathogen Citrobacter rodentium (20).

Bacterial recovery. Samples of blood, MLN, liver, and spleen were collected $2 \mathrm{~h}$ post-BT induction, weighed, minced (solid tissue), and suspended in a known volume of saline, and a $100-\mu \mathrm{L}$ aliquot was cultured on MacConkey agar plates. To confirm the identity of the aEPEC and tEPEC strains isolated from distinct organs, PCR analysis of the eae gene and serogroup identification were performed. Recovery of the HS strain was confirmed by the inability to ferment lactose (characteristic of this strain) and serogrouping.

To confirm the recovery of $E$. coli $\mathrm{R}-6$, organ suspensions were inoculated into tetracycline-containing MacConkey agar $(20 \mu \mathrm{g} / \mathrm{mL})$, and the resulting colonies were confirmed as $E$. coli by biochemical assays. Bacterial recovery from organs (CFU/g tissue) is reported as the mean $\log _{10}$ value. The median value of bacterial recovery from each compartment per gram was referred to as the BT index.

\section{Animals and groups}

Adult female Wistar-EPM rats, weighing 200-250 g, received rat chow and water ad libitum. Prior to the experiments, rats were fasted for one day and allowed free access to water. Animals were divided into 5 groups: aEPEC (N = 11), tEPEC $(\mathrm{N}=11), \mathrm{HS}(\mathrm{N}=10), \operatorname{HB} 101(\mathrm{~N}=6), \mathrm{R}-6$ $(N=6)$, and sham $(N=6)$, where saline was used instead of bacteria. For the microcirculation study, four additional animals per group were used.

The study was approved by the Research Ethics Committee of Universidade Federal de São Paulo.

\section{Bacterial translocation assay}

Animals were kept under anesthesia (intramuscular injection of $0.1 \mathrm{~mL} / 100 \mathrm{~g}$ body weight ketamine and xylazine, 4:1) during the entire procedure. An additional half-dose of 
anesthetic was given when needed. BT was induced by a midline incision, oroduodenal cannulation, inoculation of 10 $\mathrm{mL} 10^{10} \mathrm{CFU} / \mathrm{mL}$ through the catheter, and confinement of the bacteria in a segment between the duodenum and ileum using ligatures, for a period of $2 \mathrm{~h}$ (6). The inoculation of bacteria caused a transient dilatation of the small bowel, which disappeared within a short period of time.

\section{Intravital microcirculation monitoring}

After $2 \mathrm{~h}$ of the BT process, animals $(\mathrm{N}=4)$ were re-laparotomized, and the mesenteric microcirculation of the small bowel was examined with an intravital video microscope (Zeiss Axioskop-2 plus, Germany) to check for leukocyte adhesion, hemorrhage and vascular obstruction.
HS, or the sham group (Figure 1).

When bacterial recovery was examined quantitatively, tEPEC, aEPEC, and E. coli HS showed substantial translocation to MLN compared to HB101 and sham groups. Conversely, tEPEC translocation to MLN was significantly lower compared to E. coli R-6 $(\mathrm{P}<0.05)$, whereas translocation of aEPEC was as high as with the $E$. coli R-6 strain. Translocation of both aEPEC and R-6 was significantly higher compared to the HS strain. However, in systemic organs (liver and spleen) BT was significantly reduced in both EPEC groups compared to the R-6 strain $(P<0.05)$. In addition, although without any statistical differences, aEPEC translocation was always higher than that of tEPEC in MLN and liver (Figure 2). These data show that only the EPEC and R-6 strains had the ability to translocate by both the lymphatic and hematological routes. Blood cultures

\section{Tissue perfusion}

Tissue perfusion was measured in the wall of the small intestine (jejunum and ileum), in the liver and in the kidneys using a laser-Doppler flow meter (BLE-21). Measurements were made before (baseline) and $2 \mathrm{~h}$ after bacterial confinement (final). The tissue perfusion unit (TPU) of each compartment is reported as the media of TP index ( $\%$ delta) using the following formula: $\%$ delta $=($ final TPU - baseline TPU $) \times 100 /$ baseline TPU.

\section{Statistical analysis}

Analysis of variance (ANOVA), Cochran's G-test and the Fisher exact test were used and a $P$ value $\leq 0.05$ was considered to be significant.

\section{Results}

\section{tEPEC and aEPEC can translocate through the intestinal mucosa under overgrowth conditions}

Like the positive control strain R-6, the tEPEC and aEPEC strains were recovered from the MLN of all 11 animals (100\%), whereas the HS strain was recovered from 8 of 10 animals $(80 \%)$. Conversely, in the systemic organs, the percentage of tEPEC translocation was lower than that of aEPEC $(P>0.05)$, occurring in the spleen (36.4 and $45.4 \%$ ) and liver (45.5 and $72.7 \%$ ), respectively. Percentages of both tEPEC and $\mathrm{aEPEC}$ translocation to the spleen and liver were significantly lower compared to the positive control strain R-6 $(P<0.05)$. Bacteria were not found to be in the extraintestinal organs of any of the animals tested with the non-pathogenic strains E. coli HB101 and

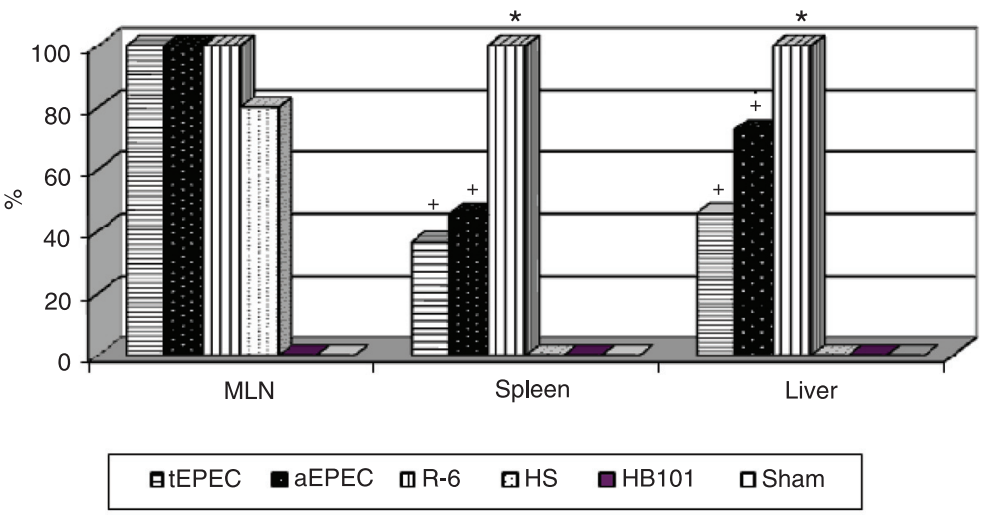

Figure 1. Percentage of animals positive for bacterial translocation (BT) to mesenteric lymph nodes (MLN), spleen and liver in a 2-h BT assay. tEPEC = typical enteropathogenic Escherichia coli; aEPEC = atypical EPEC; R-6 = positive control E. coli; $\mathrm{HS}$ and $\mathrm{HB} 101=$ non-pathogenic $E$. coli strains. $\mathrm{P}<0.05$, E. coli R-6 $\left({ }^{*}\right)$ compared with tEPEC $(+)$ and aEPEC $(+)$ in the spleen and liver (Cochran's G-test and Fisher exact test).

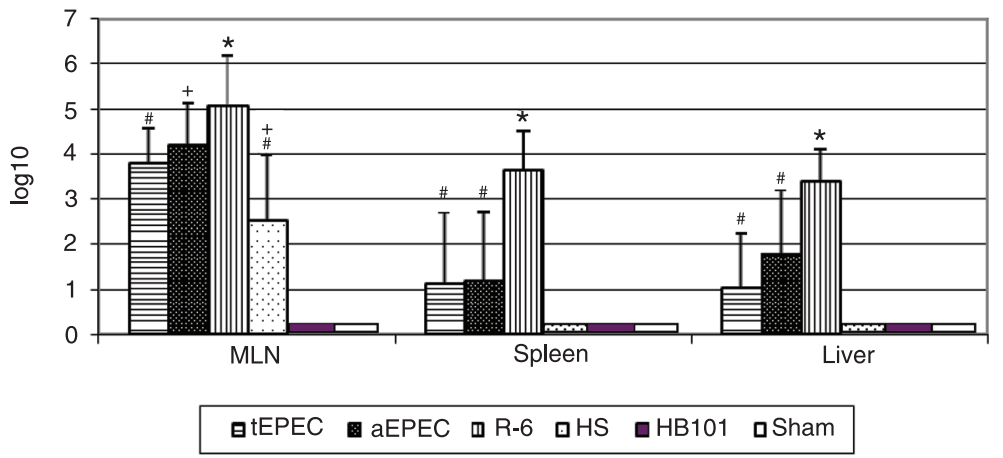

Figure 2. Bacterial recovery in all groups. Data are reported as median $\log _{10}$. Statistically significant differences $(P<0.05)$ for mesenteric lymph nodes $(M L N)$ were observed between E. coli R-6 $\left(^{*}\right)$ and tEPEC or HS (\#), and between aEPEC and HS (+). For liver and spleen, significant differences were detected between R-6 $\left(^{*}\right)$ and tEPEC or aEPEC (\#) (ANOVA and Fisher exact test). BT = bacterial translocation. See Figure 1 for explanation of abbreviations. 
were negative in all groups.

When only animals with BT to organs were considered, tEPEC and aEPEC recovery was 3.6 and $2.7 \log _{10}$ for liver and 3.4 and $3.12 \log _{10}$ for spleen, respectively. These findings were similar to those for E. coli R-6 (3.4 $\log _{10}$ for liver and $3.6 \log _{10}$ for spleen), suggesting that the differences between the EPEC groups were mostly related to the frequency (\%) of the BT event.

\section{aEPEC and tEPEC have an apparently similar} potential to induce host mesenteric microcirculation injuries compared to $E$. coli R-6

In the positive control group (R-6), after $2 \mathrm{~h}$ of the BT event, microcirculation injuries were intense and consisted of multifocal capillary hemorrhage, obstruction of low-flow venules, slower flow in high-flow venules, and increased leukocyte rolling with adhesions mainly on venule endothelial cells (Figure 3B).

BT-related microcirculation injuries of both EPEC strains were quite similar to those of E. coli R-6 (Figure $3 C$ and D). In all groups, the mesenteric arterioles and high-flow venules remained patent with normal blood flow, showing that an acute BT induction was unable to damage high-flow blood vessels. Microcirculation injuries were not seen in any negative control group.

\section{aEPEC translocation impairs tissue perfusion in a systemic manner}

Based on the findings of BT-related mesenteric mi-
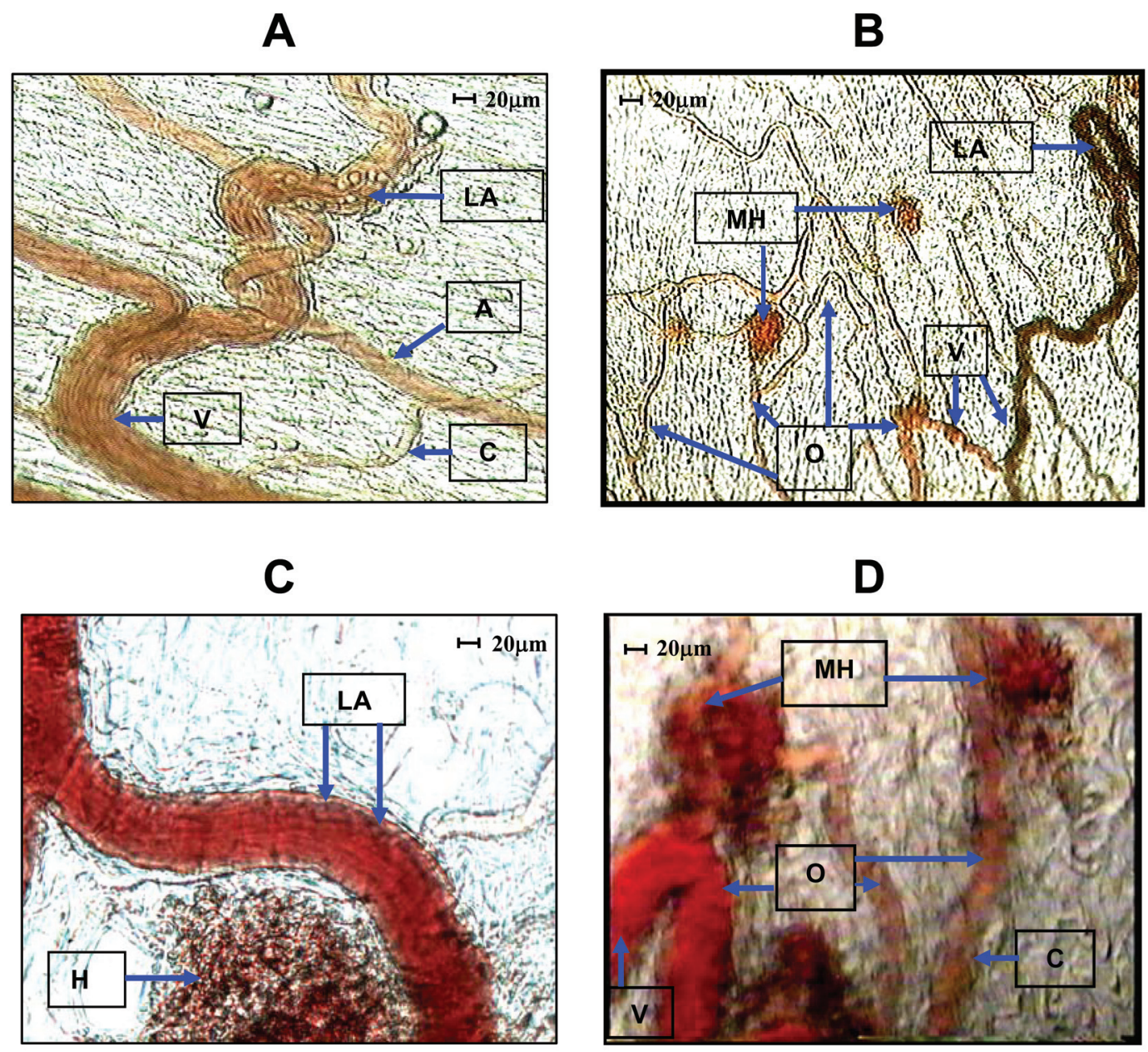

Figure 3. Mesenteric microcirculation injuries following $2 \mathrm{~h}$ of bacterial translocation (BT) as evaluated by intravital videomicroscopy. A, Negative control groups: appearance of normal microcirculation with few leukocyte adhesions $(\mathrm{LA})$ and preserved blood flow in arterioles $(\mathrm{A})$, capillaries $(\mathrm{C})$, and venules $(\mathrm{V})$. B, E. coli R-6 strain: intense leukocyte adhesion to venules, obstruction $(\mathrm{O})$ in venules and capillaries, multiple focal hemorrhages $(\mathrm{MH})$ in capillaries. $C$, tEPEC strain: increased leukocyte adhesion (LA) to venule endothelial cells and focal hemorrhage $(H)$ in capillaries. $D$, aEPEC strain: multiple focal hemorrhages in capillaries and obstructions in low-flow venules and capillaries, similar to findings for the Escherichia coli R-6 strain. See Figure 1 for explanation of groups. 
crocirculation injury, we examined if this injury could impair the tissue perfusion of the gut and of other distant systemic organs (Figure 4).

aEPEC caused similar tissue hypoperfusion in the jejunum, ileum, liver, and kidneys compared to E. coli R-6, although less severely. In contrast, tEPEC caused hypoperfusion only in the kidneys and had significantly lower effects than $E$. coli R-6 in the jejunum, ileum and liver. In addition, tEPEC-related hypoperfusion was more similar to that caused by the HB101 and HS strains, while aEPEC hypoperfusion was more similar to that caused by $E$. coli R-6. These data suggest a greater systemic inflammatory effect due to aEPEC BT, based on the broader tissue hypoperfusion seen with the aEPEC strain compared to the tEPEC strain. Nevertheless, a statistically significant difference between the effects of aEPEC and tEPEC was only seen in the jejunum.

In the sham group, the injection and confinement of saline in the gut increased final TPU in all compartments compared to baseline TPU, resulting in a positive TP index. This finding was expected on the basis of the assumption that the inoculated saline would be absorbed with a resulting increase in blood volume, with the ensuing higher tissue perfusion. Similarly, the negative BT control groups (E. coli HB101 and HS) also showed a positive TP index in the gut and liver, in addition to a nonsignificant decrease in the kidneys compared to the sham group.

\section{Discussion}

In the present study, we observed that both the tEPEC and
aEPEC strains have the capacity to translocate under conditions of a small bowel overgrowth, not only to MLN but also to systemic organs. Besides its BT potential, the aEPEC strain was even capable of causing mesenteric microcirculation injuries in addition to liver, small gut and kidney hypoperfusion. These findings were unexpected because of the consensus thatEPEC is an infectious agent restricted to the intestinal mucosa.

Remarkably, the BT capacity of aEPEC was as high as that of the positive control strain E. coli R-6, whose translocation ability has been consistently demonstrated in an in vivo rat BT model $(6-8,21)$. Furthermore, we have also shown previously that systemic tissue hypoperfusion by a BT process is mainly dependent on GALT activation by the lymphatic route of translocation while the hematological route of $\mathrm{BT}$ is more related to bacterial passage from the gut lumen into the bloodstream and further to the systemic organs (22). Furthermore, a recent kinetic study of the BT process showed that BT caused a significant and long-lasting increased release of inflammatory cytokines and lymphocytes into the gut mesenteric efferent lymph (data not shown). These findings show that the BT process is of significant relevance in the genesis of inflammation, but clinical confirmation is still lacking.

Since splanchnic hypoperfusion leading to gut tissue hypoxia and cell damage is one of the key factors related to GALT activation $(23,24)$, the aEPEC BT-associated hypoperfusion of splanchnic organs reinforces the strain's pathological potential besides its well-known diarrheagenic capacity.

If we consider only the animals with positive BT to MLN, liver and spleen, the BT index was similar for tEPEC, aEPEC and E. coli R-6. Thus, the lesser systemic hypoperfusion ef-

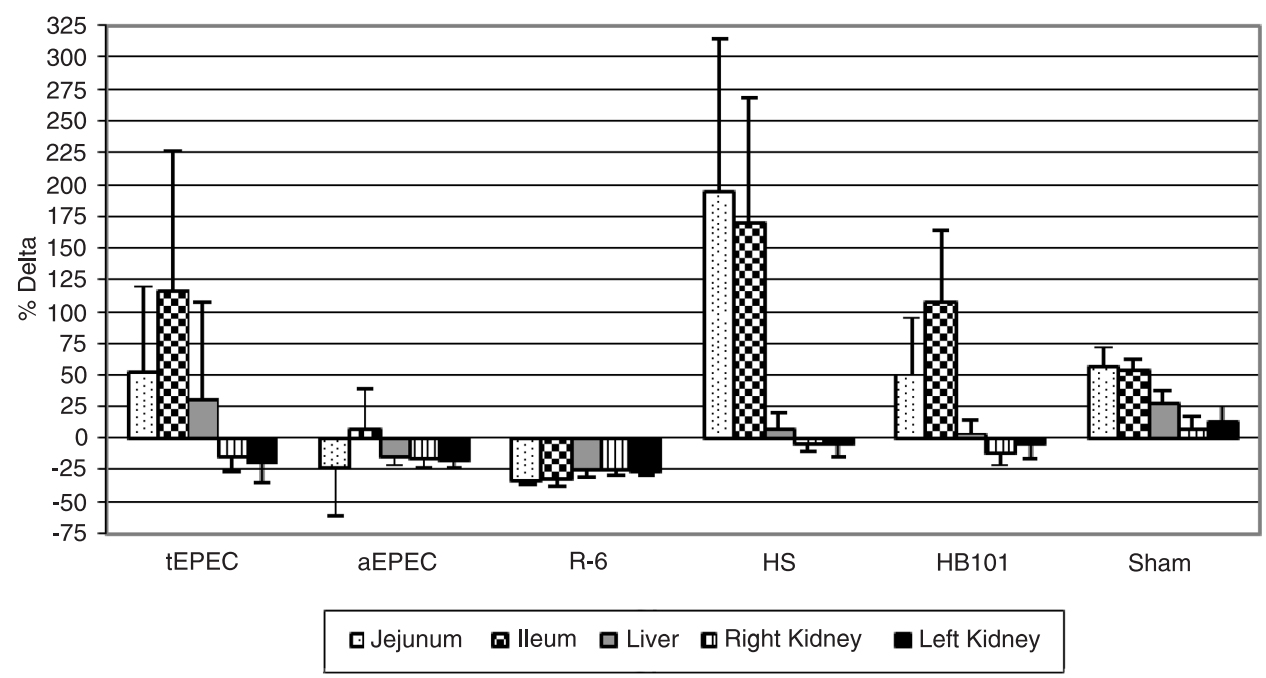

Figure 4. Tissue perfusion index of jejunum, ileum, liver, and kidneys following bacterial translocation in all groups. Data are reported as median delta \% with standard deviation. Statistical findings: In jejunum: tEPEC vs aEPEC $(P=0.048)$ or Escherichia coli R-6 $(P=0.008)$; aEPEC vs HS $(P=0.002)$; $R-6$ vs HS (0.0001). In ileum: tEPEC vs R-6 ( $\mathrm{P}=0.001)$; aEPEC vs HS $(\mathrm{P}=0.006)$; HS vs R-6 $(\mathrm{P}=0.0001)$. In liver: R-6 vs tEPEC or HS ( $\mathrm{P}=0.003)$. In right and left kidneys: $\mathrm{R}-6$ vs $\mathrm{HS}(\mathrm{P}=0.003)$. HS did not differ from the sham and HB101 groups in any organ (ANOVA and Fisher exact test). See Figure 1 for explanation of groups. 
fect of the tEPEC strain compared to E. coli R-6 and aEPEC suggests its lower systemic inflammatory potential, which could be attributed to a lower virulence potential. Studies are in progress to determine the EPEC virulence factors involved in the BT process and their systemic effects.

In the negative control groups, only E. coli HS was able to translocate to MLN, suggesting that the epithelial cell adhesion capacity of the HS strain enabled its translocation to MLN in the gut overgrowth state. However, the low amount of $\mathrm{HS}$ recovery from MLN, the absence of recovery from systemic organs, the preserved systemic organ perfusion, and mesenteric microcirculation pattern denote its limited capacity to activate the host inflammatory response.

We have recently demonstrated that the EPEC strains used in this study are able to cause AE lesions in the in vivo rabbit ileal loop model (25). Nonetheless, their ability to cause $A E$ lesions in the rat intestine and the mechanisms they use to overcome the mucosal epithelial barrier remain to be evaluated. It has already been shown that the EPEC-type three secretion system (T3SS) is required to induce loss of intestinal barrier function and to support translocation across $\mathrm{M}$ cells (26). We are currently testing an isogenic T3SS mutant of the aEPEC strain used in this study to determine whether effector proteins injected by

\section{References}

1. Deitch EA. Bacterial translocation of the gut flora. J Trauma 1990; 30: S184-S189.

2. Berg RD. Bacterial translocation from the gastrointestinal tracts of mice receiving immunosuppressive chemotherapeutic agents. Curr Microbiol 1983; 8: 285-292.

3. Schaedler RW, Dubs R, Costello R. Association of germfree mice with bacteria isolated from normal mice. J Exp Med 1965; 122: 77-82.

4. Cohen J. The immunopathogenesis of sepsis. Nature 2002; 420: 885-891.

5. Leaphart CL, Tepas JJ III. The gut is a motor of organ system dysfunction. Surgery 2007; 141: 563-569.

6. Koh IH, Silva RM. Novel in vitro small intestinal graft model for study of bacterial translocation in the rat. Transplant Proc 1996; 28: 2667-2668

7. Koh IH, Menchaca-Diaz JL, Farsky SH, Siqueira AF, RuizSilva M, Juliana P, et al. Injuries to the mesenteric microcirculation due to bacterial translocation. Transplant Proc 2002; 34: 1003-1004.

8. Koh IHJ, Menchaca-Diaz JL, Bugni GM, Watanabi AY, Silva FJP, Ruiz-Silva M, et al. The role of the mesenteric lymph on microcirculation injury during bacterial translocation. Cri Care 2003; 7 (Suppl 3): 29.

9. Menchaca-Diaz JL, Silva RM, Figueiredo LFP, Bugni GM, Watanabe AY, Silva FJP, et al. Bacterial translocation consequential to intestinal bacterial overgrowth provokes aggravation of mortality by sepsis. Cri Care 2003; 7 (Suppl 3): 28.

10. Gatt M, Reddy BS, MacFie J. Review article: bacterial translocation in the critically ill - evidence and methods of this system could play a role in our model of BT.

Since the microcirculation changes were seen within a short BT event, in the case of long-lasting gut overgrowth conditions an enduring EPEC BT event could cause greater pathological damage.

Although experimental results are far from clinical events, the present findings suggest that diarrheagenic EPEC strains have the further potential to promote extraintestinal effects.

Our findings demonstrate for the first time the capability of an aEPEC strain and a tEPEC strain to translocate and impair the systemic microcirculation in an experimental study. However, other EPEC strains have to be investigated to find out if these phenomena are also associated with other EPEC strains.

\section{Acknowledgments}

Dr. A. Leyva provided English editing of the manuscript. Research supported by CNPq (Edital Universal \#473503/2006-9) and PRONEX (MCT/CNPq/FAPERJ \#E26/171.526/2006) awarded to T.A.T. Gomes. F.C. Moreira received a scholarship from FAPESP (\#04/14713-0).

prevention. Aliment Pharmacol Ther 2007; 25: 741-757.

11. Kaper JB, Nataro JP, Mobley HL. Pathogenic Escherichia coli. Nat Rev Microbiol 2004; 2: 123-140.

12. Trabulsi LR, Keller R, Tardelli Gomes TA. Typical and atypical enteropathogenic Escherichia coli. Emerg Infect Dis 2002; 8: 508-513.

13. Gomes TA, Irino K, Girao DM, Girao VB, Guth BE, Vaz TM, et al. Emerging enteropathogenic Escherichia coli strains? Emerg Infect Dis 2004; 10: 1851-1855.

14. Chen HD, Frankel G. Enteropathogenic Escherichia coli: unravelling pathogenesis. FEMS Microbiol Rev 2005; 29: 83-98.

15. Kaper JP. Defining EPEC. In: Proceedings of the International Symposium on Enteropathogenic Escherichia coli (EPEC). Rev Microbiol 1996; 27: 130-133.

16. Dean P, Kenny B. Intestinal barrier dysfunction by enteropathogenic Escherichia coli is mediated by two effector molecules and a bacterial surface protein. Mol Microbiol 2004; 54: 665-675.

17. Shifflett DE, Clayburgh DR, Koutsouris A, Turner JR, Hecht GA. Enteropathogenic E. coli disrupts tight junction barrier function and structure in vivo. Lab Invest 2005; 85: 13081324.

18. Levine MM, Caplan ES, Waterman D, Cash RA, Hornick RB, Snyder MJ. Diarrhea caused by Escherichia coli that produce only heat-stable enterotoxin. Infect Immun 1977; 17: 78-82.

19. Gannon VP, Rashed M, King RK, Thomas EJ. Detection and characterization of the eae gene of Shiga-like toxinproducing Escherichia coli using polymerase chain reaction. 
J Clin Microbiol 1993; 31: 1268-1274.

20. Deng W, Li Y, Vallance BA, Finlay BB. Locus of enterocyte effacement from Citrobacter rodentium: sequence analysis and evidence for horizontal transfer among attaching and effacing pathogens. Infect Immun 2001; 69: 6323-6335.

21. Koh IH, Montero EF, Keller R, Abreu MC, Goldenberg S, Silva RM. Can the portal vein be a conduit for bacterial translocation? Transplant Proc 1996; 28: 2671.

22. Koh IH, Liberatore AM, Menchaca-Diaz JL, Ruiz-Silva M, Vilela-Oliveira L, Watanabe AY, et al. Bacterial translocation, microcirculation injury and sepsis. Endocr Metab Immune Disord Drug Targets 2006; 6: 143-150.

23. Clark JA, Coopersmith CM. Intestinal crosstalk: a new paradigm for understanding the gut as the "motor" of critical illness. Shock 2007; 28: 384-393.

24. Magnotti LJ, Deitch EA. Burns, bacterial translocation, gut barrier function, and failure. J Burn Care Rehabil 2005; 26 : 383-391.

25. Moreira FC, Vieira MA, Ferreira AJ, Girao DM, Vaz TM, Rosa $\mathrm{AC}$, et al. Escherichia coli strains of serotype O51:H40 comprise typical and atypical enteropathogenic $E$. coli strains and are potentially diarrheagenic. J Clin Microbiol 2008; 46 : $1462-1465$

26. Martinez-Argudo I, Sands C, Jepson MA. Translocation of enteropathogenic Escherichia coli across an in vitro $\mathrm{M}$ cell model is regulated by its type III secretion system. Cell Microbiol 2007; 9: 1538-1546. 